\title{
Linx
}

Revue des linguistes de l'université Paris X Nanterre

$13 \mid 2020$

Collocations et traditions discursives

\section{Au sujet de la traduction des comparaisons stéréotypées selon la grammaire de construction. Le cas de « alt com un sant Pau » dans La plaça del Diamant et ses traductions.}

\section{Pedro Ivorra Ordines}

\section{OpenEdition}

\section{Journals}

Édition électronique

URL : http://journals.openedition.org/linx/3903

DOI : 10.4000/linx.3903

ISSN : 2118-9692

Éditeur

Presses universitaires de Paris Nanterre

Référence électronique

Pedro Ivorra Ordines, « Au sujet de la traduction des comparaisons stéréotypées selon la grammaire de construction. Le cas de « alt com un sant Pau » dans La plaça del Diamant et ses traductions. », Linx [En ligne], 13 | 2020, mis en ligne le 30 mai 2020, consulté le 01 juin 2020. URL : http:// journals.openedition.org/linx/3903; DOI : https://doi.org/10.4000/linx.3903

Ce document a été généré automatiquement le 1 juin 2020

Département de Sciences du langage, Université Paris Ouest 


\title{
Au sujet de la traduction des comparaisons stéréotypées selon la grammaire de construction. Le cas de « alt com un sant Pau » dans $L a$ plaça del Diamant et ses traductions.
}

\author{
Pedro Ivorra Ordines
}

\section{Introduction}

1 La phraséologie, et plus spécifiquement les comparaisons stéréotypées, sont un outil fondamental pour les locuteurs d'une langue au moment de donner aux énoncés l'expressivité adéquate à la situation. Néanmoins, pour les personnes qui sont en train d'apprendre une nouvelle langue, maitriser ce genre d'expressions représente un véritable défi, car souvent celles-ci comportent des particularités linguistiques et culturelles qui sont plutôt propres à une culture donnée. De la même manière, pour le traducteur, faire face à la traduction de ce genre d'expressions est aussi un défi de taille, étant donné que les problèmes selon Corpas «no se originan únicamente en la "incompetencia" de los traductores en este tema, sino que depende también [...] de las diferencias existentes entre los universos fraseológicos de las lenguas ${ }^{1} »$ (Corpas 2003 : 215).

2 L'étude que nous proposons ci-dessous a pour but d'étudier le domaine des comparaisons stéréotypées considérées comme étant des phénomènes propres à la discipline de la phraséologie et en utilisant l'approche de la grammaire de construction par la même occasion. Après avoir mené à bien l'étude d'une construction sur le plan intralinguistique, notre intention est d'observer comment cette construction a été traduite vers l'anglais, vers l'espagnol et vers le français, l'objectif étant de décrire ce que les traducteurs ont fait dans chacune des traductions. 
3 Cet article comportera d'abord une section de nature théorique. Nous y exposerons dans un premier temps les caractéristiques des comparaisons stéréotypées selon la discipline de la phraséologie, en tant que ressources linguistiques chargées d'intensifier un fait avec un contenu dénotant. Nous présenterons dans un second temps les particularités qui définissent ce genre de constructions selon l'approche de la Grammaire de Construction -dorénavant GC-. Une fois établies les bases théoriques de notre objet d'étude, nous observerons, à travers une description détaillée, comment a été effectuée la traduction de la comparaison stéréotypée "alt com un san Pau " (littéralement: 'haut/grand comme un saint Paul') (Rodoreda 1962: 174), selon une approche constructionnelle, en soulignant l'un des concepts centraux de cette approche : la non-modularité.

\section{Cadre théorique}

\subsection{La discipline de la phraséologie}

4 La discipline de la phraséologie a pour objet l'étude des unités phraséologiques dorénavant, UP. Parmi les UP qui constituent cette discipline, nous nous concentrerons sur le cas particulier des comparaisons stéréotypées; pour ce faire nous prendrons pour référence les travaux de García-Page (cf. 2008a, 2008b et 2009) en langue espagnole puisqu'il n'y a pas d'études détaillées en catalan. Dans ces travaux, on mène une description minutieuse de la comparaison stéréotypée, toujours en opposition avec la comparaison libre, d'un point de vue syntaxique, sémantique et pragmatique.

5 La comparaison stéréotypée est une particularité linguistique que nous, les locuteurs, nous avons à notre disposition, et grâce à laquelle nous exprimons une qualité au degré superlatif. Par conséquent, sa fonction principale est l'intensification. Tout comme la plupart des UP, tels les refrains, les locutions, etc., la comparaison stéréotypée "se trata de una fórmula comprimida del saber enciclopédico; [...] de ahí su extraordinaria fuerza argumentativa, en gran medida su ingrediente de enunciado enfático ${ }^{2}$ » (GarcíaPage 2008b: 144).

6 Afin de bien cerner les caractéristiques de notre objet d'étude, nous opposerons la comparaison stéréotypée à la comparaison libre ou grammaticale pour savoir quelles sont les caractéristiques propres à ce genre d'expression.

7 En ce qui concerne l'aspect syntaxique, et en prenant l'exemple « alt com un san Pau ", la comparaison stéréotypée face à la comparaison libre se caractérise, entre autres, par:

- La possibilité de changer une comparaison d'égalité en une comparaison de supériorité sans aucune répercussion sémantique : alt com un sant Pau = més alt que un sant Pau (littéralement : 'haut/grand comme un saint Paul = 'plus haut/grand qu'un saint Paul). En revanche, dans la comparaison libre il y a des modifications de sens: Pedro es tan alto como Juan $\neq$ Pedro es más alto que Juan [littéralement: 'Pedro est aussi haut/grand que Juan' $\neq$ ‘Pedro est plus haut/ grand que Juan'].

- La possibilité de paraphraser la comparaison stéréotypée, ce qui n'a pas lieu avec la comparaison libre. Par conséquent, on aura alt com un sant Pau = muy alto/altísimo (littéralement : 'haut/grand comme un saint Paul' = très haut/grand/grandissime).

- Les éléments qui forment la comparaison stéréotypée ne peuvent être échangés. Par conséquent, alt com un sant Pau $\neq$ sant Pau com alt (littéralement : 'haut/grand comme un saint 
Paul' ₹ 'saint Paul comme haut/grand'). En revanche, cet échange est possible dans le cas d'une comparaison libre, bien qu'il y ait des modifications de sens. Par conséquent, Pedro es más alto que Juan $\neq$ Juan es más alto que Pedro (littéralement : 'Pedro est plus haut que Juan' $\neq$ 'Juan est plus haut que Pedro').

En ce qui concerne l'aspect sémantique, et en prenant le même exemple, la comparaison stéréotypée face à la comparaison libre se caractérise, entres autres, par :

- une corrélation qui s'établit entre la base de la comparaison et le prototype, de sorte qu'il y a une implication lexicale orientée vers le prototype. De ce fait, l'intensification caractéristique principale de ce genre d'expression- ne s'obtient pas uniquement moyennant des procédures grammaticales, mais également moyennant des déductions et des suppositions (García-Page 2009: 341), comme par exemple avec alt com un sant Pau (littéralement : 'haut/grand comme un saint Paul'), qui représente le degré de hauteur/ grandeur maximum.

- la possibilité de comprendre une comparaison stéréotypée sans connaître le référent, dans ce cas précis, sant Pau ('saint Paul'). Néanmoins, cela fonctionne sémantiquement parlant, car le référent qualifie culturellement la caractéristique.

- la particularité de fixation des comparaisons stéréotypées nous permet de comprendre et d'utiliser les comparaisons, même si le référent n'existe pas ou n'est plus d'actualité. C'est le cas pour notre exemple, où le référent n'existe plus, mais pour lequel la comparaison continue à s'utiliser grâce à une fixation.

- La valeur culturelle du prototype. Dans ce cas, sant Pau ('saint Paul') représente en catalan le prototype culturel de la hauteur/grandeur maximum et, par conséquent, c'est le représentant extralinguistique culturel de la hauteur/grandeur, au degré superlatif.

La nature compositionnelle, très peu idiomatique et à la signification prévisible ${ }^{3}$ a mené de nombreux linguistes à nier le statut de schéma phraséologique aux comparaisons stéréotypées et, par conséquent, à les exclure du centre de l'univers phraséologique et à les relier aux collocations ${ }^{4}$.

\subsection{L'approche de la grammaire de construction dans les études de traduction}

10 Les études de traduction et de linguistique entretiennent une « love-hate relationship » (Rojo \& Ibarretxe-Antuñano $2013: 3$ ) depuis toujours. D'un côté, cette relation d'amourhaine a été marquée par une attirance mutuelle: les chercheurs en traduction ont trouvé dans la linguistique des concepts qu'ils appliquaient à la traduction et parallèlement les linguistes ont trouvé dans la traduction une source très profitable d'exemples pour l'enseignement des langues et les études contrastives de langues. De l'autre côté, cette attirance s'est vue également durement touchée : les linguistes ont jugé la traduction comme étant une activité linguistique de seconde catégorie, considérée comme inappropriée pour l'enseignement des langues pendant de longues années, tandis que les chercheurs dans le milieu de la traduction ont réagi face à l'attitude des linguistes en déclarant la linguistique incapable d'expliquer les aspects cognitifs et culturels de la traduction.

11 La fin des années soixante-dix et le début des années quatre-vingt ont vu le passage d'une approche prescriptiviste vers une autre de nature descriptive dans les études de traduction, même si toutes les approches alors utilisées ont été critiquées parce que dans chacune d'entre elles les auteurs adoptaient une approche contrastive où le 
problème central était l'équivalence au niveau des mots ou des phrases. L'utilisation de phrases décontextualisées a entrainé de nombreux doutes parmi les théoriciens du moment, qui manifestèrent le besoin d'un changement d'approche, qui s'est développé entre les années quatre-vingt et quatre-vingt-dix avec le «cultural turn» (Rojo \& Ibarretxe-Antuñano 2013: 5), soit le tournant culturel. Il y a deux approches principales qui ont prédominé lors de cette période : l'approche fonctionnaliste (avec différents modèles proposés par House, Nida ou Nord) et l'approche socioculturelle (avec la Théorie des Polysystèmes de Toury ou l'École de la Manipulation de Hermans). Néanmoins, à partir des années quatre-vingt-dix, les études académiques se sont intéressés à la linguistique cognitive en tant qu'approche théorique pour résoudre toutes les questions restantes des décennies précédentes.

Actuellement, les études de traduction ont le désir de décrire la traduction en tant que processus cognitif et tendent à adopter une approche interdisciplinaire pouvant contribuer à décrire ce processus de diverses manières. Par consequent «if any linguistic theory has something to contribute to translation studies must be one in which meaning acquires a central role, and one in which language is naturally linked to cognitive processes ${ }^{5}$ » (Rojo \& Valenzuela 2013: 284). Parmi les possibles théories candidates, nous proposons dans cet article l'approche linguistique de la grammaire de construction dans les études de traduction ${ }^{6}$.

13 Le principe fondamental de la GC est que les constructions traditionnelles sont les unités basiques du langage, qui ont une signification, indépendamment de la phrase (Goldberg 1995: 6). Les théoriciens qui adoptent cette approche vis-à-vis du langage visent à caractériser et à décrire la totalité des structures qui le constituent. Toutes les analyses constructionnistes ont en commun l'idée que les constructions sont des paires apprises, constituées d'une forme associée à une signification, où les morphèmes, mots, locutions et phrases sont inclus. Les constructions peuvent se définir comme suit :

[a]ny linguistic pattern is recognized as a construction as long as some aspect of its form or function is not strictly predictable from its component parts or from other constructions recognized to exist. In addition, pattern are stored as constructions even if they are fully predictable as long as they occur with sufficient frequency. (Goldberg 2006:5) ${ }^{7}$

Comprendre la construction comme «form-meaning pairs ${ }^{8}$ » (Goldberg 1995 : 71), soit des unions de forme et signification, est quelque chose qui est intimement lié à un concept de grande importance de la GC: la non-modularité, un concept que nous essayerons d'expliquer plus en détail ci-après.

Par non-modularité, nous observons que «sound, syntactic form, meaning, and function are not to be specified in separate, autonomous modules ${ }^{9}$ " (Fried \& Östman 2004: 14), mais plutôt que la grammaire est composée d'unions de forme et de signification, ce qui nous permet d'offrir une description holistique des constructions. De cette façon, nous comprenons que les constructions fonctionnent comme des schémas pour les locuteurs/auditeurs, en leur fournissant les directives et les conditions préalables sémantiques et pragmatiques pour savoir quand et comment il est possible d'utiliser ces schémas (Fried \& Östman 2004 : 14). Par conséquent, et pour visualiser l'idée de construction, nous nous imaginons un bloc que le locuteur a en tête, dans lequel il a des informations de nature différente.

Il est important de préciser à partir de l'image précédente qu'il n'existe pas de niveaux ou catégories entre les différentes propriétés qui composent les constructions. Les 
constructions sont considérées comme étant des clusters ou bundles d'informations et la grammaire comme un répertoire de tous ces clusters $^{10}$.

\subsection{Les comparaisons stéréotypées selon l'approche constructionnelle}

Comme nous l'avons signalé à la fin de la section concernant la discipline de la phraséologie, les caractéristiques propres à ce genre d'expressions faisaient que ces expressions en question étaient reléguées au second plan. Néanmoins, grâce à l'approche constructionnelle, la dichotomie entre centre et périphérie a pu être dépassée, ainsi que celle existant entre les structures prototypiques et les structures productives, pour ainsi arriver à un continuum entre le pôle grammatical et le pôle lexical. Par conséquent :

[1]as construcciones fraseológicas ocupan en este continuum un lugar intermedio por estar parcialmente saturadas en sus constituyentes. Las unidades que se acercan más al polo léxico presentan un alto grado de saturación en sus constituyentes léxicos, al contrario de lo que sucede con las construcciones más próximas al polo gramatical, que además son productivas, menos restrictivas y más frecuentes ${ }^{11}$. (Mellado sous presse)

Ce genre de construction se caractérise par les aspects suivants (Mellado 2012:2-3, $2015: 2)$ :

- Il s'agit de constructions qui ont une signification unique, plus ou moins abstraite selon la construction.

- Certains des composants de la structure syntaxique sont déjà fixes sémantiquement parlant, tandis que d'autres se sont remplis lexicalement dans le discours (slots).

- Les slots sont renouvelés dans le discours moyennant des lexèmes correspondant à un profil sémantique concret, ce qui indique que l'occupation de ces slots est sujette à des restrictions sémantiques. Le degré de restriction sémantique de chacun des slots libres dépend de chaque construction.

- Les constructions ont une signification pragmatique spécifique.

\section{Analyse du cas : "alt com un sant Pau » et ses traductions}

19 L'exemple dont nous allons parler ci-après appartient à l'ouvrage La plaça del Diamant, un roman de l'écrivaine catalane Mercè Rodoreda (1908-1983), traduite dans plus de trente langues différentes, parmi lesquelles le chinois, le néerlandais, le japonais, le grec, le norvégien, entre beaucoup d'autres (Mallafrè 2010:69) et considérée de ce fait comme éune des écrivaines contemporaines à la plus grande envergure internationale $\mathrm{du} \mathrm{XX}^{\mathrm{ème}}$ siècle. Nous avons choisi cet ouvrage parce que l'auteure s'y démarque de par son style ${ }^{12}$ très élaboré, où l'on distingue un certain savoir-faire au niveau de la rédaction des dialogues et de la construction des scènes.

De plus, dans ce style élaboré avec minutie prédomine le langage parlé, un registre qui se caractérise par l'usage abondant de répétitions, d'expressions toutes faites, de phrases coordonnées ou de comparaisons. Parmi les ressources que nous avons mentionnées, nous nous concentrerons sur les comparaisons stéréotypées, et plus particulièrement sur la comparaison « alt com un sant Pau » : 


\begin{tabular}{|c|c|}
\hline $\begin{array}{l}\text { Identifiant } \\
\text { CAT }^{13} \\
\text { Chapitre } \\
24 \\
\text { page } 173\end{array}$ & $\begin{array}{l}\text { Fins que a l'últim em va demanar que el perdonés per haver-me vingut a explicar } \\
\text { històries d'ell, que un home se les ha de saber passar tot sol, però que em coneixia feia } \\
\text { tant de temps, i que em coneixia tan bé, que li semblava com si fos germana seva, i } \\
\text { quan va dir que em considerava com si fos germana seva se'm va arrencar a plorar i } \\
\text { em vaig espantar molt. Era la primera vegada que veia plorar un home alt com un sant } \\
\text { Pau i amb els ulls blaus }{ }^{14} \text {. }\end{array}$ \\
\hline $\begin{array}{l}\text { Identifiant } \\
\text { EN1 } \\
\text { Chapitre } \\
24, \\
\text { page } 108\end{array}$ & $\begin{array}{l}\text { And then in the end he asked me would I forgive him for coming here telling stories of } \\
\text { himself, that a man should be able to go through things by himself, but he knew me so } \\
\text { long now, and he knew me so well, that I seemed to him like his own sister, and when } \\
\text { he said he thought of me as his own sister, he burst into tears and gave me an even } \\
\text { bigger fright. It was the first time I ever saw a man cry, and he a great big giant of a } \\
\text { man with his blue eyes. }\end{array}$ \\
\hline $\begin{array}{l}\text { Identifiant } \\
\text { EN2 } \\
\text { Chapitre } \\
24, \\
\text { page } 109\end{array}$ & $\begin{array}{l}\text { Till finally he asked me to forgive him for coming to me with his troubles when a man } \\
\text { has to know how to deal with them alone, but he knew me so well and for such a long } \\
\text { time that I was like a sister to him and when he said I was like a sister he started } \\
\text { crying and it scared me a lot. It was the first time I'd seen a man cry who was as tall as } \\
\text { Saint Paul and had blue eyes. }\end{array}$ \\
\hline $\begin{array}{l}\text { Identifiant } \\
\text { EN3 } \\
\text { Chapitre } \\
24 \\
\text { page } 104\end{array}$ & $\begin{array}{l}\text { Until he apologised for coming and burdening me with his problems, that a man has to } \\
\text { face his heartaches by himself, but he'd known me for so long and so well he thought } \\
\text { of me as a sister, and when he said that he burst into tears and that frightened me. It } \\
\text { was the first time I'd seen a man cry, a man who was tall as St Paul and blue-eyed into } \\
\text { the bargain. }\end{array}$ \\
\hline $\begin{array}{l}\text { Identifiant } \\
\text { ES } \\
\text { Chapitre } \\
24 \\
\text { page } 127\end{array}$ & $\begin{array}{l}\text { Hasta que al final me pidió que le perdonase por haberme venido a contar sus } \\
\text { historias, que un hombre tiene que saber arreglárselas solo, pero que me conocía hacía } \\
\text { tanto tiempo, y que me conocía tan bien, que le parecía como si yo fuese una hermana } \\
\text { suya, y cuando dijo que me consideraba como si fuese hermana suya se me echó a } \\
\text { llorar y me asusté mucho. Era la primera vez que veía llorar a un hombre, alto como un } \\
\text { San Pablo y con los ojos azules. }\end{array}$ \\
\hline $\begin{array}{l}\text { Identifiant } \\
\text { FR } \\
\text { Chapitre } \\
24, \\
\text { page } \\
122-123\end{array}$ & $\begin{array}{l}\text { À la fin il m'a demandé de le pardonner d'être venu me raconter ses histoires, un } \\
\text { homme doit savoir supporter ses malheurs tout seul, mais ça faisait si longtemps qu'il } \\
\text { me connaissait, et il me connaissait si bien qu'il avait l'impression que j'étais sa sœur, } \\
\text { et en me disant qu'il me considérait comme sa soeur il a éclaté en sanglots et j'ai eu } \\
\text { vraiment peur. C'était la première fois que je voyais pleurer un homme fort comme un } \\
\text { Turc et avec les yeux bleus. }\end{array}$ \\
\hline
\end{tabular}

21 En ce qui concerne les aspects formels de la construction [ADJ + com $\left.+\mathrm{DET}_{[u n]}+\mathrm{N}\right]$ nous observons que la comparaison stéréotypée dispose d'un élément référentiel ou tertium comparationis (alt) qui, dans ce cas précis, est un adjectif et qui présente une qualité commune au comparé et au comparant ; une particule comparative (com, dans le cas de la comparaison d'égalité), et un comparant ou prototype ${ }^{15}$ (sant Pau 'saint Paul'), qui est la source d'inspiration de la comparaison. De plus, il y a un comparé, même s'il ne fait pas partie des composants de la construction et qu'il a une fonction syntaxique de sujet, qui dans ce cas serait le personnage de Mateu. 

cas a une structure comparative (avec un adverbe comparatif), où le deuxième élément de la comparaison renforce l'expressivité du premier élément. Par conséquent, dans ce cas précis, le deuxième élément (sant Pau 'saint Paul') intensifie le premier élément (alt 'grand') au degré superlatif. De plus, l'intensification de l'adjectif est corroborée par la définition de cette comparaison stéréotypée : "alt com un sant Pau SA, es diu d'una persona molt alta " ["grand/haut comme un saint Paul SA, se dit d'une personne de grande taille » (Notre traduction)] (Espinal $2006: 144$ ).

D'un point de vue pragmatique, la particule comparative com ('comme') est un indicateur de la fonction d'intensification de la signification, qui en même temps indique la volonté de capter ou d'attirer l'attention du lecteur. Moyennant ces expressions, l'auteur ne prétend pas comparer deux éléments de la réalité extralinguistique, mais plutôt attirer l'attention du lecteur pour l'impliquer dans l'évaluation de l'énoncé et mettre l'accent sur l'intensité d'un fait avec un contenu dénotatif. Ainsi donc nous observons que l'auteur tente de mettre l'accent sur le fait que, bien qu'il s'agisse d'un homme grand et costaud, Mateu a également des sentiments et qu'il les exprime au travers de ses larmes; ce qui, aux yeux de la protagoniste, peut être considéré comme paradoxal. En outre, toujours sur le plan pragmatique, mais en nous concentrant sur des aspects purement extralinguistiques, nous pouvons dire que l'origine de cette expression est basée sur une tradition d'origine médiévale ${ }^{16}$. Lors de certaines fêtes auxquelles participaient les différents corps de métiers et confréries de la ville avec leurs drapeaux et bannières respectifs, les forgerons défilaient normalement derrière un personnage représentant Saint Paul qui portait l'épée de la ville, et celle-ci étant de grandes dimensions, c'était l'homme le plus corpulent qui avait pour habitude de la porter (Sugranyes 2000: 80).

Dans la première traduction vers l'anglais, et d'un point de vue formel, nous observons que la construction de $\left[\mathrm{ADJ}+\mathrm{com}+\mathrm{DET}_{[u n]}+\mathrm{N}\right] \mathrm{du}$ texte d'origine a été modifiée en $\left[\mathrm{DET}_{[\mathrm{a}]}+\mathrm{ADJ} 1+\mathrm{ADJ} 2+\mathrm{N}\right]$, bien que cela ne soit pas la conséquence d'une restriction constructionnelle. En d'autres termes, l'anglais dispose de comparaisons stéréotypées pour exprimer la hauteur/grandeur au degré superlatif (comme par exemple as tall as a giraffe $^{17}$ 'grand comme une girafe'), même si la traductrice n'a pas utilisé ce genre de constructions dans ce cas précis. De plus, nous observons que la collocation d'adjectifs great big est fréquente ${ }^{18}$ en anglais et que, d'un point de vue sémantique, ils serviraient d'intensificateurs du substantif qu'ils précèdent. Néanmoins, ce substantif peut ajouter de l'information qui n'est pas présente dans le texte d'origine car l'usage du substantif giant ('géant') peut entrainer des connotations liées aux traditions mythiques ou pseudo-historiques dans la fiction romantique ${ }^{19}$. Finalement, d'un point de vue pragmatique, nous observons qu'il y a une parfaite correspondance avec la construction du texte d'origine obtenue grâce aux deux adjectifs de la construction, même s'il y a des pertes en ce qui concerne les aspects extralinguistiques.

En ce qui concerne la deuxième et la troisième traductions vers l'anglais, et d'un point de vue formel, elles ressemblent à la construction du texte d'origine, car dans les deux cas ils ont opté pour une comparaison d'égalité $[(a s)$ ADJ + as + N]. De ce fait, le traducteur a décidé d'utiliser la technique de la traduction littérale dans le texte d'arrivée. Néanmoins, si nous regardons la construction de près, nous pouvons observer que dans le deuxième cas le premier élément comparatif de la comparaison, facultatif en anglais, a été omis. En outre, nous observons que, dans ces deux cas, on n'a 
pas utilisé de déterminant, et le mot "Saint» a été écrit avec une majuscule initiale. D'un point de vue sémantique, cela peut prêter à confusion, car l'usage d'une majuscule et l'absence d'un déterminant font qu'il est fait référence au saint, qui, comme nous l'avons dit précédemment, était de petite taille. Il convient également de penser à la référence concernant la cathédrale londonienne ${ }^{20}$, de sorte que de ce point de vue il y aurait une correspondance sur le plan sémantique avec la construction du texte d'origine. Finalement, d'un point de vue pragmatique, nous voyons que la traduction fonctionne, car le contexte en fin de compte nous fait comprendre comment est la personne, même si la traduction peut paraître étrange aux yeux des lecteurs parce qu'elle n'est pas fréquente ${ }^{21}$.

En ce qui concerne la traduction vers l'espagnol, d'un point de vue formel, le traducteur dans ce cas précis a opté pour une comparaison d'égalité [ADJ + como + $\left.\mathrm{DET}_{[u n]}+\mathrm{N}\right]$, avec une traduction littérale de la construction du texte d'origine, bien que le traducteur disposait de comparaisons stéréotypées fixes dans la langue pour exprimer le degré de hauteur/grandeur maximum comme "como un pino " ('comme un sapin', Seco et al. $2004: 801$ ). D'un point de vue sémantique et pragmatique, à cause d'une nette influence du catalan (nous voyons que le traducteur a utilisé la technique du calque), nous observons que dans les deux cas il y a une correspondance avec la comparaison stéréotypée du texte d'origine. Néanmoins, nous constatons qu'il s'agit d'une comparaison fréquente dans des sources journalistiques, probablement en raison du contact entre l'espagnol et le catalan.

En ce qui concerne la traduction vers le français, nous observons que, d'un point de vue formel, il y a une correspondance avec la construction du texte d'origine, car dans les deux cas le traducteur a opté pour une comparaison d'égalité avec la même structure syntaxique $\left[\mathrm{ADJ}+\right.$ como $\left.+\mathrm{DET}_{[u n]}+\mathrm{N}\right]$. Néanmoins, le français dispose d'unités phraséologiques pour indiquer le degré maximum de hauteur/grandeur, comme " armoire normande» ou "armoire à glace " ${ }^{22}$. D'un point de vue sémantique, le traducteur a choisi de souligner une autre qualité du personnage, car dans le texte d'origine c'est la hauteur/grandeur qui est soulignée, tandis que dans la traduction l'accent est porté sur la force (fort: «LOC. Fort comme un Turc ${ }^{23}$ : très fort." Le Petit Robert). Malgré les différences au niveau sémantique, nous pouvons observer que la traduction fonctionne au niveau pragmatique, même si dans la traduction c'est la force qui prime et non la taille. En effet, comme dans le texte d'origine, mais en mettant l'accent sur une autre qualité, est exprimé ce qui semblerait tout aussi irréel à Natàlia : le fait qu'une personne aussi forte soit capable de se mettre à pleurer face aux épreuves de la vie. Néanmoins, il y a une perte de la valeur culturelle du texte d'origine, même s'il y a aussi des bénéfices culturels.

\section{Notre bilan}

28 À partir de cette analyse nous nous sommes rendu compte que, normalement, ce sont les constructions "périphériques » qui posent problème aux traducteurs et, donc, qui sont les plus intéressantes "since they often involve complex configurations of properties, including pragmatic ones, which are not clustered together in the target language ${ }^{24} »$ (Szymańska 2011a: 220). Par conséquent, l'approche constructionnelle nous permet de voir la difficulté de trouver un équivalent aussi bien vis-à-vis de la forme que de la signification dans la langue d'arrivée. En d'autres termes, l'approche 
constructionnelle nous permet de voir quels sont les gains, losses et ungains ${ }^{25}$ pendant le processus de traduction. De cette façon, nous pouvons observer que dans la première traduction vers l'anglais, le traducteur a décidé de chercher un équivalent au niveau sémantique et pragmatique, car nous obtenons la hauteur/grandeur du personnage et nous constatons l'incrédulité de la protagoniste face à cet homme qui pleure. Néanmoins, l'utilisation de "giant " ('géant') peut entrainer certaines nuances qui ne se trouvent pas dans le texte d'origine. De plus, et de la même manière que dans le cas des autres traductions, sauf peut-être dans le cas de l'espagnol, la valeur culturelle de la construction du texte d'origine se perd. En ce qui concerne les deuxième et troisième traductions vers l'anglais, nous y observons que l'importance a été accordée à la forme, de sorte que le référent culturel soit transmis au lecteur du texte traduit. La signification (niveau sémantique) et l'intention (niveau pragmatique) peuvent être équivalentes à la construction du texte d'origine grâce au contexte, même si la construction peut sembler être étrange aux yeux des lecteurs étant donné qu'il s'agit d'une traduction littérale. En ce qui concerne la traduction vers l'espagnol, nous observons que nous sommes face au même cas que dans les deux traductions précédentes, donnant lieu également à une impression étrange pour les lecteurs étant donné qu'il s'agit de constructions qui ne s'utilisent pas fréquemment en langue espagnole. Finalement, en ce qui concerne la traduction vers le français, nous observons que le traducteur a donné plus d'importance à la forme qu'à l'intention, ce qui nous donne la même construction au niveau syntaxique et l'intention est réussie, car la protagoniste s'étonne ici aussi des pleurs de Mateu. Cependant nous observons que sur le plan sémantique, le traducteur a choisi une construction avec une signification complètement différente de celle de la construction du texte d'origine, et, en outre, les connotations de ces constructions sont différentes de celles de la construction de départ.

\begin{tabular}{|c|c|c|c|c|}
\hline & $\begin{array}{l}\text { NIVEAU } \\
\text { FORMEL }\end{array}$ & NIVEAU SÉMANTIQUE & NIVEAU PRAGMATIQUE & $\begin{array}{l}\text { TECHNIQUE } \\
\text { DE } \\
\text { TRADUCTION }\end{array}$ \\
\hline CAT & $\begin{array}{l}{[\mathrm{ADJ}+} \\
\mathrm{COM}^{+} \\
\mathrm{DET}_{[\mathrm{UN}]}+ \\
\mathrm{N}]\end{array}$ & $\begin{array}{l}\text { Renvoie à la propriété } \\
\text { d'être une personne } \\
\text { très grande. }\end{array}$ & $\begin{array}{l}\text { Intensification et connotations } \\
\text { culturelles liées à la tradition }\end{array}$ & \\
\hline EN1 & $\begin{array}{l}{[\mathrm{ADJ}+} \\
\mathrm{ADJ}+\mathrm{N}]\end{array}$ & personne très grande & $\begin{array}{l}\text { Cela fonctionne, même si le mot 'giant' } \\
\text { ('géant') peut comporter des } \\
\text { implications liées aux traditions } \\
\text { mythiques ou pseudo-historiques dans } \\
\text { la fiction romantique. De plus, il y a une } \\
\text { perte de la valeur culturelle. }\end{array}$ & Paraphrase \\
\hline EN2 & $\begin{array}{l}{[\mathrm{AS}+\mathrm{ADJ}} \\
+\mathrm{AS}+\mathrm{N}]\end{array}$ & \multirow{3}{*}{$\begin{array}{l}\text { Même s'il s'agit de } \\
\text { constructions qui ne } \\
\text { sont pas recueillies } \\
\text { dans les sources } \\
\text { documentaires (en } \\
\text { provoquant une non- } \\
\text { naturalité et une }\end{array}$} & \multirow{2}{*}{$\begin{array}{l}\text { Malgré cette sensation d'être face à } \\
\text { quelque chose d'étrange dans ces trois } \\
\text { cas, cela n'empêche pas de comprendre } \\
\text { l'énoncé comme étant une } \\
\text { intensification. En d'autres termes, la } \\
\text { signification de la construction }\end{array}$} & $\begin{array}{l}\text { traduction } \\
\text { littérale }\end{array}$ \\
\hline EN3 & $\begin{array}{l}{[\mathrm{ADJ}+\mathrm{AS}} \\
+\mathrm{N}]\end{array}$ & & & $\begin{array}{l}\text { traduction } \\
\text { littérale }\end{array}$ \\
\hline & & & $\begin{array}{l}\text { signification de la construction } \\
\text { comparative en tant qu'intensification } \\
\text { est si forte que l'on peut comprendre la }\end{array}$ & \\
\hline \multicolumn{2}{|c|}{ Linx, 13 | 2020} & $\begin{array}{l}\text { sensation étrange chez } \\
\text { le lecteur), la } \\
\text { signification se déduit } \\
\text { grâce au contexte. }\end{array}$ & $\begin{array}{l}\text { contexte s'y prête. Cet argument } \\
\text { renforce la vision constructionnelle, } \\
\text { car la forme et le contenu sont liés. En }\end{array}$ & \\
\hline
\end{tabular}




\begin{tabular}{|c|c|c|c|c|}
\hline ES & $\begin{array}{l}{[\mathrm{ADJ}} \\
\mathrm{COMO} \\
\mathrm{DET}_{[\mathrm{UN}]} \\
\mathrm{N}]\end{array}+$ & & & calque \\
\hline FR & $\begin{array}{l}{[\mathrm{ADJ}+} \\
\mathrm{COMME}^{+}+ \\
\mathrm{DET}_{[\mathrm{UN}]}+ \\
\mathrm{N}]\end{array}$ & $\begin{array}{l}\text { Renvoie à la propriété } \\
\text { d'être une personne } \\
\text { très forte. }\end{array}$ & $\begin{array}{l}\text { Cela fait ressortir la force de Mateu, } \\
\text { nous voyons que cela fonctionne même } \\
\text { s'il s'agit d'une personne forte } \\
\text { (physiquement parlant), car il peut se } \\
\text { mettre à pleurer. Il y a une perte de la } \\
\text { valeur culturelle du texte d'origine, } \\
\text { même s'il y a aussi des bénéfices } \\
\text { culturels. }\end{array}$ & $\begin{array}{l}\text { comparaison } \\
\text { stéréotypée }\end{array}$ \\
\hline
\end{tabular}

Selon cette analyse, nous avons pu démontrer le potentiel d'un des composants de l'approche constructionnelle pour décrire l'un des problèmes auxquels les traducteurs doivent se confronter quand ils se trouvent face à ce genre de constructions. Concrètement, cette première étude a permis de souligner l'importance de l'interaction et la continuité des connaissances linguistiques et extralinguistiques dans le processus de traduction, ainsi que l'inclusion de la dimension pragmatique du langage, qui sont les piliers centraux de l'approche constructionnelle du langage. Ceci dit, nous estimons qu'il serait très intéressant de continuer à explorer la traduction des comparaisons stéréotypées, en utilisant comme cadre théorique des concepts ou des points de vue propres à l'approche constructionnelle du langage.

\section{BIBLIOGRAPHIE}

CORPAS, Gloria (2003) : Diez años de investigación en fraseología: análisis sintácticosemánticos, contrastivos y traductológicos. Madrid : Lingüística Iberoamericana.

CROFT, William (2007) : "Construction Grammar". The Oxford Handbook of Cognitive Linguistics, ed. by Dirk GEERAERTS \& Hubert CUYCKENS. New York : Oxford University Press, 463-508.

ESPINAL, Maria Teresa (2006) : Diccionari de Sinònims i Frases Fetes. Barcelona : Universitat de Barcelona. Servei de Publicacions.

FILLMORE, Charles / KAY, Paul / O'CONNOR, Mary (1988) : "Regularity and Idiomaticity in Grammatical Constructions: The Case of Let Alone”. Language 64, 3 : 501-538.

FRIED, Mirjam \& ÖSTMAN, Jan-Ola (2004) : "Construction Grammar: A thumbnail sketch". Construction Grammar in a cross-language perspective, ed. by Mirjam FRIED and Jan-Ola ösTMAN. Amsterdam and Philadelphia : John Benjamins Publishing Company, 11-86.

GARCÍA-PAGE, Mario (2008a) : Introducción a la fraseología española. Estudio de las locuciones. Barcelona : Anthropos. 
GARCÍA-PAGE, Mario (2008b) : “La comparativa de intensidad: la función del estereotipo”. Verba 35 : 143-178.

GARCÍA-PAGE, Mario (2009) : "Propiedades sintácticas de la comparativa estereotipada en español". Romanistisches Jahrbuch 59 : 339-360.

GOLDBERG, Adele (1995) : A Construction Grammar Approach to Argument Structure. Chicago :

University of Chicago Press.

GOLDBERG, Adele (2006) : Constructions at Work: the nature of generalizations at language. Oxford : Oxford University Press.

KAY, Paul (2002) : "An Informal Sketch of a Formal Architecture of Construction Grammar". Grammars $5: 1-19$.

KOIKE, Kazumi (2005) : “Colocaciones complejas en el español actual”. Fraseología contrastiva con ejemplos tomados del alemán, español, francés e italiano, ed. by Ramón ALMELA, Estanislao RAMÓN \& Gerd wотјАк. Murcia : Servicio de Publicaciones Universidad de Murcia, 169-184.

KOIKE, Kazumi (2008) : “Locuciones verbales con base colocacional”. Revista de Filología 26 : 75-94. LE PETIT ROBERT (2017). Paris : Le Robert. Resource en ligne [Dernier access 21/12/2018].

MALLAFRÈ, Joaquim (2010) : “Les traduccions de l'obra de Mercè Rodoreda". Congrés Internacional Mercè Rodoreda: ACTES, Barcelona 1-5 d'octubre de 2008, dir. per Joaquim MOLAS. Barcelona : Sociedad Estatal de Conmemoraciones Culturales / Institut d'Estudis Catalans, Fundació Mercè Rodoreda, 69-81.

MELLADO, Carmen (2012) : “Las comparaciones fijas en alemán y español: algunos apuntes contrastivos en torno a la imagen fija". Linred: Lingüística en Red $10: 1-32$.

MELLADO, Carmen (2015) : “Antiphrasis-based Comparative Constructional Idioms in Spanish". Journal of Social Sciences $11: 111-127$.

MELLADO, Carmen (sous presse) : “Esquemas fraseológicos y construcciones fraseológicas en el contínuum léxico-gramática”. Clases y categorías en la fraseología española, ed. por Carsten SINNER, Esteban MONTORO \& Encarnación TABARES. Leipzig : Leipziger Universitätsverlag.

OXFORD ENGLISH DICTIONARY (2001). Oxford : Oxford University Press. Online resource [Last time accessed 21/12/2018].

ROJO, Ana \& IBARRETXE-ANTUÑANO, Iraide (2013) : "Cognitive Linguistics and Translation Studies: Past, present and future". Cognitive Linguistics and Translation: Advances in Some Theoretical Models and Applications, ed. por Ana Rojo \& Iraide IBARRETXE-ARTUÑANO. Berlín and Boston : De Gruyter, 3-30.

Rojo, Ana \& VAlenzuela, Javier (2013) : "Constructing meaning in translation : The role of construction in translation problems". Cognitive Linguistics and Translation : Advances in Some Theoretical Models and Applications, ed. por Ana Rojo \& Iraide IBARRETXE-ARTUÑANO. Berlín and Boston : De Gruyter, 283-310.

SECO, Manuel / ANDRÉs, Olimpia / RAMOS, Gabino (2004) : Diccionario fraseológico documentado del español actual. Locuciones y modismos. Madrid : Aguilar.

SOBRÉ, Josep Miquel (1973) : "L’artifici de La plaça del Diamant, un estudi lingüístic". In memoriam Carles Riba (1959-1969). Esplugues de Llobregat : Institut d'Estudis Helllènics / Ariel, 363-375. 
SUGRANYES, Josep (2000) : Garbellada de refranys. Aires de serè en la nostra parla. Valls: Edicions Cossetània.

SZYMAŃSKA, Izabela (2011a) : “Construction Grammar as a Framework for Describing Translation: A Prolegomenon”. New Perspectives in Language, Discourse and Translation Studies, ed. by Miroslaw PAWLAK \& Jakub BIELAK. Berlín : Springer, 215-224.

SZYMAŃSKA, Izabela (2011b) : Mosaics. A Construction-Grammar-Based Approach to Translation. Varsovia : Semper.

\section{Corpus}

RODOREDA, Mercè (1962) : La plaça del Diamant. Barcelona : Bromera.

RODOREDA, Mercè (1967) : The pigeon girl, trans. by Eda O'SHIEL. London : André Deutsch.

RODOREDA, Mercè (1980) : The Time of the Doves, trans. by David ROSENTHAL. New York : Graywolf Press.

RODOREDA Mercè (2006) : La Place du Diamant, trad. par Bernard LESFARGUES. Paris : Gallimard.

RODOREDA, Mercè (2007) : La Plaza del Diamante, trad. por Enrique soRDO. Barcelona : Ediciones Orbis S.A.

RODOREDA, Mercè (2013) : In Diamond Square, trans. by Peter BUSH. London : Virago.

\section{NOTES}

1. " ne trouvent pas uniquement leur origine dans "l'incompétence" de traducteurs à ce sujet, mais cela dépend également [...] de différences qui existent entre les univers phraséologiques des langues » (Notre traduction).

2. «est une formule comprimée du savoir encyclopédique; [...] c'est de là que vient son extraordinaire force argumentative, en grande partie son statut d'ingrédient de l'énoncé emphatique " (Notre traduction).

3. La signification de ce genre de structure est prévisible, selon Mellado, pour deux raisons : «la estructura $<\mathrm{X}$ es más $(\mathrm{Z})$ que $Y>$ es indicadora en sí misma de una intensificación semántica, de un componente elativo" ["la structure $<\mathrm{X}$ est plus ( $\mathrm{Z}$ ) que $\mathrm{Y}>$ indique en elle-même une intensification sémantique, d'un élément élatif' (notre traduction)] et «los prototipos o comparatos suelen poseer valores semántico-culturales prefijados extrafraseológicamente, que anticipan y anuncian el significado fraseológico de las comparaciones con un mismo prototipo » ['les prototypes et les comparants ont normalement des valeurs sémantico-culturelles préétablies extraphraséologiquement, qui anticipent et annoncent la signification phraséologique des comparaisons avec un même prototype' (Notre traduction)] (Mellado 2012: 2).

4. Voir Koike $(2005,2008)$, qui défend qu'il s'agit de collocations complexes avec une base collocationnelle et un collocatif. De plus, García-Page affirme que cela « apoya nuestra visión de las colocaciones como productos de la Sintaxis y no como unidades de la Fraseología » ['soutient notre façon de voir les collocations comme étant des produits de la syntaxe et non comme étant des unités de la phraséologie' (Notre traduction)] (García-Page 2008a: 38).

5. 'si une théorie linguistique peut contribuer de quelque manière que ce soit aux études de traduction, il doit y en avoir une dans laquelle le sens acquiert un rôle central et une dans laquelle le langage est naturellement lié aux processus cognitifs' (Notre traduction).

6. Pour cette étude, nous suivons la proposition théorique-appliquée de Szymańska (2011a, 2011b). 
7. 'N'importe quel modèle linguistique est reconnu comme étant une construction tant qu'un certain aspect de sa forme ou de sa fonction n'est pas strictement prévisible d'après ses composants ou d'autres constructions dont l'existence est reconnue. En outre, les modèles sont enregistrés comme étant des constructions même s'ils sont complètement prévisibles tant qu'ils apparaissent assez fréquemment' (Notre traduction).

8. 'assemblages de forme et de sens' (Notre traduction).

9. 'le son, la forme syntactique, la signification et la fonction ne doivent pas être spécifiés séparément, comme des modules autonomes' (Notre traduction).

10. De la même façon que nous le voyons sur le schéma, Fillmore et al. affirment que "a large part of a language user's competence is to be described as a repertory of clusters of information including, simultaneously, morphosyntactic patterns, semantic interpretation principles to which these are dedicated, and, in many cases, specific pragmatic functions in whose service they exist » [('une grande partie de la compétence linguistique d'un utilisateur est décrite comme étant un répertoire de groupes d'informations qui inclut, simultanément, des modèles morphosyntactiques, des principes d'interprétation sémantique auxquels elles se consacrent et, dans de nombreux cas, des fonctions pragmatiques spécifiques au service desquelles elles existent.') (Notre traduction)] (Fillmore et al. $1988: 534$ ).

11. 'dans ce continuum, les constructions phraséologiques occupent une place intermédiaire parce qu'elles sont partiellement saturées dans leurs composants. Les unités qui se rapprochent le plus du pôle lexical présentent un haut degré de saturation dans leurs composants lexicaux, contrairement à ce qui se passe avec les constructions qui sont plus proches du pôle grammatical, qui en outre sont plus productives, moins restrictives et plus fréquentes.' (Notre traduction).

12. Voir Sobré (1973) pour une analyse approfondie du style de l'ouvrage.

13. Nous utiliserons la nomenclature suivante pour les ouvrages utilisés dans cette analyse : CAT (La plaça del Diamant), EN1 (The pigeon girl), EN2 (The Time of the Doves), EN3 (In Diamond Square), ES (La plaza del Diamante) et FR (La place du Diamant).

14. Le contexte dans lequel la comparaison stéréotypée est utilisée est le suivant : Mateu et Griselda ont eu des problèmes de couple et étant donné que Griselda a commencé à travailler, elle doit laisser leur fille chez ses parents. Mateu s'inquiète de ce qui pourrait lui arriver. Il souffre énormément et décide d'aller raconter cette situation à Natàlia, en qui il a grandement confiance étant donné tous les moments passés ensemble lors de la rénovation de la sa maison. Néanmoins, après lui avoir tout raconté, il regrette qu'elle se soit inquiétée et se met à pleurer, ce qui choque Natàlia, car elle pense qu'il est impossible qu'un homme aussi grand et costaud que Mateu puisse pleurer. De plus, le fait qu'il s'agisse d'un homme rend la chose encore plus irréelle à ses yeux, il s'agit en effet du premier homme qu'elle voit pleurer.

15. Selon la terminologie de García-Page (2008b).

16. Si nous considérons les données historiques recueillies jusqu'à ce jour, l'apôtre saint Paul était de petite taille et chauve. De ce fait, nous pouvons observer que la comparaison stéréotypée utilise un déterminant indéfini, ce qui veut dire qu'il est fait référence à l'image d'un saint, et non au saint en question; c'est à dire qu'il n'est pas utilisé en tant que nom propre, mais comme un nom commun.

17. Oxford English Dictionaries.

18. Great : «Preceding a partly synonymous adjective, passing into a simple intensifier. Frequently in great big. Now also preceding an intensifier, as bloody. Now colloq » Oxford English Dictionary. De ce fait, dans une recherche faite dans le corpus Timestamped JSIweb corpus 2014-2018 de Sketch Engine un total de 27881 entrées de la collocation « great big » sont comptabilisées.

19. Giant: "One of the supposed beings in human form but of superhuman stature, who occur frequently in mythic or pseudo-historical traditions and in romantic fiction. In Greek mythology,

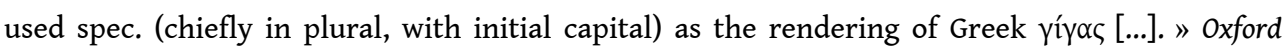
English Dictionary. 
20. Nous pourrions penser qu'il pourrait s'agir de la cathédrale de Londres, car elle fut considérée comme étant l'un des bâtiments les plus hauts de la ville jusqu'en 1962 (https:// goo.gl/hqzqj).

21. La recherche des constructions «tall as St Paul» et «tall as Saint Paul» dans Sketch Engine (concrètement dans le corpus Timestamped JSIweb corpus 2014-2018), nous renvoie uniquement à un résultat de la construction «tall as St Paul » : « and when finished comprised of 1.2 million pages and if stacked would be twice as tall as St Paul's Cathedral in London " ('une fois terminé, il contient 1,2 million de pages et, si nous les empilons, cela serait deux fois plus grand que la cathédrale Saint-Paul de Londres') (Notre traduction).

22. «FIG. et FAM. personne de carrure impressionnante. » Le Petit Robert; bien que dans ce cas nous serions en train de mettre encore plus l'accent sur la corpulence de la personne en question. 23. «De nos jours, un Turc n'est jamais qu'un être humain comme un autre. Et même s'il y a des Turcs qui détiennent des records du monde en haltérophilie, rien ne semble justifier qu'on qualifie plus de fort un Turc qu'un Grec, un Monégasque ou un Chinois. Mais il ne faut pas oublier l'histoire de la Turquie. Avant que ce pays ne devienne ce qu'il est aujourd'hui, il y a eu l'Empire ottoman bâti par un peuple de guerriers à coups de conquêtes en Europe, en Afrique et en Asie. Ces combattants turcs ou ottomans impressionnaient par leur force, leur courage et aussi leur brutalité, leur cruauté. C'est ainsi qu'au XVII ${ }^{\mathrm{e}}$ et XVIII ${ }^{\mathrm{e}}$ siècle, le Turc symbolisait l'incroyant, l'ennemi brutal. On disait d'ailleurs de quelqu'un de rude et de sans pitié qu'il était "un vrai Turc" et traiter quelqu'un "à la turque", c'était le traiter sans ménagement. L'expression est née au milieu du XV $\mathrm{XV}^{\mathrm{e}}$ siècle, un peu après la prise de Constantinople (l'ancienne Byzance et l'Istanbul d'aujourd'hui) par les troupes du sultan Mehmet II en 1453.» (Les expressions françaises décortiquées, https://goo.gl/dKpH8M).

24. 'car elles impliquent souvent des configurations complexes de propriétés, y compris pragmatiques, qui ne sont pas regroupées dans la langue cible' (Notre traduction).

25. Terme créé par Szymańska pour faire référence à « unwelcome side-effects (for instance stylistic inconsistency or unintended associations) brought into the TT by some linguistic choices" [('effets secondaires indésirables (incohérences stylistiques ou associations involontaires, par exemple) survenus dans la traduction suite à certains choix linguistiques') (Notre traduction)] (Szymańska 2011b: 127).

\section{RÉSUMÉS}

Cet article a pour but de faire une étude contrastive et traductologique des comparaisons stéréotypées en utilisant l'approche constructionnelle. Dans cette étude, notre intention est d'utiliser une approche linguistique, utilisant les principes de la grammaire de construction comme fondement théorique, pour mener à bien une analyse descriptive de la traduction des comparaisons stéréotypées. Nous pourrons ainsi observer quelles sont les implications du choix d'une construction particulière entre les différentes traductions sur le plan formel, sémantique, pragmatique et culturel.

This paper presents a contrastive and translational analysis of stereotyped comparisons using a constructionist approach. To that end, our main aim is to use a linguistic approach, by taking into consideration the constructionist tenets as theoretical framework, in order to descriptively analyse the translation of stereotyped comparisons. With this study, we will be able to see what 
entails choosing a specific construction from a formal, semantic, pragmatic and cultural point of view.

INDEX

Keywords : Construction Grammar, stereotyped comparisons, translation, contrastive and translational analysis

Mots-clés : grammaire de construction, comparaisons stéréotypées, traduction, étude contrastive et traductologique

\section{AUTEUR}

\section{PEDRO IVORRA ORDINES}

Universitat Pompeu Fabra, Barcelona 